\title{
Family History of Autoimmune Disease in Patients with Aicardi-Goutières Syndrome
}

\author{
Johanna L. Schmidt, ${ }^{1,2}$ Ivana Olivieri, ${ }^{3}$ Jodie M. Vento, ${ }^{1}$ Elisa Fazzi, ${ }^{4}$ \\ Heather Gordish-Dressman, ${ }^{2}$ Simona Orcesi, ${ }^{3}$ and Adeline Vanderver ${ }^{1,2}$ \\ ${ }^{1}$ Department of Neurology, Children's National Medical Center, Washington, DC 20010, USA \\ ${ }^{2}$ Center for Genetic Medicine Research, Children's National Medical Center, Washington, DC 20010, USA \\ ${ }^{3}$ Unit of Child Neurology and Psychiatry, C. Mondino National Institute of Neurology Foundation, Pavia 27100, Italy \\ ${ }^{4}$ Department of Child and Adolescent Neuropsychiatry, University of Brescia, Brescia 25123, Italy
}

Correspondence should be addressed to Simona Orcesi, simona.orcesi@mondino.it

Received 17 June 2012; Accepted 1 October 2012

Academic Editor: Timothy B. Niewold

Copyright ( $) 2012$ Johanna L. Schmidt et al. This is an open access article distributed under the Creative Commons Attribution License, which permits unrestricted use, distribution, and reproduction in any medium, provided the original work is properly cited.

Purpose. The purpose of this study was to explore anecdotal evidence for an increase in the prevalence of autoimmune diseases in family members of patients with Aicardi-Goutières syndrome (AGS). Methods. Pedigrees of patients and controls were analyzed using chi-square and logistic regression to assess differences in reports of autoimmune disease among family members of cases and controls. Data was collected at Children's National Medical Center in Washington, DC, USA and at the International AicardiGoutières Syndrome Association Scientific Headquarters, C. Mondino National Institute of Neurology in Pavia, Italy. Results. The number of individuals with reported autoimmune disease is significantly related to having a family member with AGS $\left(\chi^{2}=6.25\right.$, $P=0.01) ; 10 \%(35 / 320)$ of relatives of patients with AGS had a reported autoimmune disease diagnosis compared to 5\% (18/344) of relatives of controls. There was a greater percent of maternal relatives of patients with AGS reporting autoimmune disease $(14.6 \%)$, compared to controls $(6.8 \%)$, with the association being statistically significant. The association was not significant for paternal relatives. Conclusion. The prevalence of autoimmune disease in relatives of children with AGS is significantly increased compared to controls. More research is needed to better understand this association.

\section{Introduction}

Aicardi-Goutières syndrome (AGS) is a heritable neurologic disorder with an immune basis. Patients most typically present early in life with increased cerebrospinal fluid (CSF) interferon alpha and markers of inflammation, elevated liver enzymes, thrombocytopenia, intracranial calcifications and leukoencephalopathy. Patients with AGS usually demonstrate severe neurologic dysfunction and life-long disability.

The immune basis of AGS was originally suspected by Aicardi and Goutières, as a persistent CSF pleocytosis was seen in affected infants. Infants presented with what appeared to be a congenital infection [1], and reports of elevations of CSF IFN $\alpha[2]$ and neopterin $[3,4]$ further suggested that an immune process was at play. However, it was not until the identification of AGS related mutations in nucleases, including TREX1 and the three constituent subunits of RNase H2, as well as a nonnuclease, SAMHD1, that the relationship between innate cellular immunity and AGS began to be more fully understood.

Discovery of the genes associated with AGS allowed for further definition of the phenotype. Most patients with AGS were found to have homozygous or compound heterozygous changes in these genes. AGS was also found to be allelic with Cree encephalitis and an inherited infantile systemic lupus erythematosus [5-8]. Heterozygous mutations in AGS related genes have also been found in rare patients with systemic lupus erythematosus (SLE) $[9,10]$ and familial chilblain lupus (FCL) [11-13], which are both autoimmune disorders. Studies in large populations of SLE patients suggest that TREX1 single nucleotide polymorphisms may also be related to neurologic manifestations and the presence 
of autoantibodies [10]. Additionally, mutations in TREX1 have been reported in patients with retinal vasculopathy with cerebral leukodystrophy (RVCL) [14].

Of the five genes currently known to be associated with AGS, four, TREX1 and RNASEH2A-C, encode proteins that have nucleic acid metabolizing functions, or nucleases. The fifth gene SAMHD1, encodes a protein that while not specifically a nuclease is thought to degrade nucleic acid precursors [15] and is upregulated by immune-stimulatory DNA [16]. Mutations in these genes are thought to result in the accumulation of endogenous nucleic acids. Growing evidence suggests that this accumulation provokes a type 1 interferon response that results in the development of pathogenic cytokines and autoantibodies that target the brain and other organs [17].

Anecdotal observation suggests an increase in the prevalence of various autoimmune diseases in the family history of children diagnosed with AGS. However, despite the evidence of AGS related mutations in some individuals from SLE populations, this has never been explored in families of AGS affected persons. The purpose of this study was to investigate this anecdotal observation to determine if there is an increase in prevalence of autoimmune disease in families who have children with a diagnosis of AGS, as compared to control families.

\section{Materials and Methods}

To investigate the possible increase in prevalence of autoimmune disease in family members of children with AGS, pedigrees were analyzed from families with AGS and controls who either had a definitive diagnosis other than AGS or who had unsolved (i.e., undiagnosed) leukodystrophies without any features of AGS (including clinical and neuroradiological findings). AGS cases were defined by having consistent clinical features (elevated alpha interferon and white blood cells in CSF, central nervous system calcifications, and leukoencephalopathy and no other identifiable etiology) and by confirmed mutations in any of the known AGS related genes.

Autoimmune disease is a broad term and, for the purposes of this study, was considered to include autoimmune thyroiditis leading to hypothyroidism, and other autoimmune thyroid dysfunction, inflammatory bowel disease, type I diabetes, rheumatoid arthritis, multiple sclerosis, Raynaud syndrome, psoriasis, scleroderma, Addison disease, and Kawasaki disease, [18-20] as well as the above mentioned autoimmune diseases (i.e., systemic lupus erythematosus and familial chilblain lupus) already known to be associated with AGS related genes.

The presence of autoimmune disease was investigated in case and control families using semistructured interviews to inquire about autoimmune disease generally and about the presence of specific autoimmune diseases (lupus, thyroid dysfunction, inflammatory bowel disease, type I diabetes, rheumatoid arthritis, multiple sclerosis, and autoimmune skin disorders). Data was collected between September 2008 and October 2011. Pedigrees for AGS subjects were drawn during AGS family clinic/conferences in 2008 and 2011 at Children's National Medical Center, or during clinical encounters in Washington DC or in Pavia, Italy. Pedigrees for control subjects were drawn during clinical encounters in Washington, DC by the same staff as performed AGS pedigrees. Pedigrees were excluded if there was insufficient information from one side of the family or if the patient was adopted and family history information was limited. Consanguineous families were included in both cases and controls. Pedigrees were drawn by genetic counselors or a pediatric neurologist with special expertise in biochemical genetics and leukodystrophy.

Data collection was part of a larger data collection effort within the European NIMBL (Nuclease Immune Mediated Brain and Lupus-like conditions) project. Italian families who participated to this study were enrolled in NIMBL in collaboration with the International Aicardi Goutieres Syndrome Association (IAGSA). United States families were enrolled in NIMBL in collaboration with the Myelin Disorders Bioregistry Project. The project was approved by the Institutional Review Board at Children's National Medical Center and the ethical review boards at the Child Neurology and Psychiatry Unit at the C. Mondino National Institute of Neurology, Pavia, Italy.

The prevalence of autoimmune disease was investigated all relatives combined, in parents only, and in second degree relatives only. In this study, half-siblings of parents were also considered to be second-degree relatives and were included in the analysis. Siblings and half-siblings of patients were not included in the analysis, as most of them were too young to have exhibited any features of autoimmune disease and may or may not develop autoimmune disease in the future.

Chi-square and/or logistic regression were used to assess differences between overall relatives, between maternal relatives and paternal relatives, between mothers and fathers, and between second-degree relatives in cases and controls.

\section{Results}

Pedigrees of families of children with AGS $(N=31)$ were compared with control pedigrees of families of children without AGS $(N=31)$. The US site collected family history information on 17 of the AGS families, while the Italian group collected family history on 14 families. Pedigrees were collected in a prospective fashion.

Controls were all from the Myelin Disorders Bioregistry at Children's National Medical Center in Washington, DC. Controls were patients who either had a clinical or molecular diagnosis other than AGS, or had an undetermined diagnosis but without features of AGS. Control diagnoses included Alexander disease, metachromatic leukodystrophy (MLD), hypomyelination with hypogonadotrophic hypogonadism and hypodontia $(4 \mathrm{H})$ syndrome, mucopolysaccharidosis IIIc, multiple sulfatase deficiency, congenital cytomegalovirus (CMV), acute disseminated encephalomyelitis (ADEM), and peroxisomal disorders.

Comparisons of autoimmune disease diagnoses were assessed in relatives of AGS patients and control patients using chi-square and logistic regression (Tables 1 and 2). 
TABLE 1: History of autoimmune disease in parents of cases and controls.

\begin{tabular}{|c|c|c|c|c|c|}
\hline Characteristic & AGS cases & Control & OR & $P$ value & $95 \% \mathrm{CI}$ \\
\hline \multicolumn{6}{|c|}{ Maternal history of AI (yes/no) } \\
\hline No & $26(83.9 \%)$ & $28(90.3 \%)$ & 1.00 & & \\
\hline Yes & $5(16.1 \%)$ & $3(9.7 \%)$ & 1.75 & 0.453 & $0.39-8.27$ \\
\hline \multicolumn{6}{|c|}{ Paternal history of AI (yes/no) } \\
\hline No & $30(96.8 \%)$ & $31(100.0 \%)$ & 1.00 & & \\
\hline Yes & $1(3.2 \%)$ & $0(0.0 \%)$ & - & $0.313^{*}$ & - \\
\hline \multicolumn{6}{|c|}{ Maternal and/or paternal history of AI (yes/no) } \\
\hline No & $25(80.7 \%)$ & $28(90.3 \%)$ & 1.00 & & \\
\hline Yes & $6(19.4 \%)$ & $3(9.7 \%)$ & 2.24 & 0.288 & $0.51-9.91$ \\
\hline
\end{tabular}

${ }^{*} P$ value from chi-squared test due to zero control subjects with a paternal history of AI.

TABLE 2: History of autoimmune disease in second degree relatives of cases and controls.

\begin{tabular}{|c|c|c|c|c|c|}
\hline Characteristic & AGS cases & Control & OR & $P$ value & $95 \% \mathrm{CI}$ \\
\hline \multicolumn{6}{|c|}{ All second degree relatives both matrilineal and patrilineal with history of AI (yes/no) } \\
\hline No & $11(35.5 \%)$ & $19(61.3 \%)$ & 1.00 & & \\
\hline Yes & $20(64.5 \%)$ & $12(38.7 \%)$ & 2.88 & 0.044 & $1.03-8.07$ \\
\hline
\end{tabular}

First, a chi-square test was performed to assess differences in reports of autoimmune diseases in the two groups. The number of relatives with reported autoimmune disease is significantly related to having a family member with AGS $\left(\chi^{2}=\right.$ $6.25, P=0.01)$ : there were 320 total relatives of patients with AGS, and 35 (10.9\%) had reported autoimmune disease diagnoses, compared to $5.2 \%(18 / 344)$ controls. Thus, the prevalence of autoimmune disease in AGS families was more than twice that of the control families.

Next, a chi-square calculation was performed on maternal versus paternal relatives. There was a greater percent of maternal relatives with reported autoimmune disease $(24 / 164$, or $14.6 \%)$ compared to controls $(12 / 176$, or $6.8 \%)$, and this association was statistically significant $(P=0.03)$. For paternal relatives, the association between having diagnosis of an autoimmune disease and having a relative with AGS was not significant.

Using logistic regression, when looking at all first degree relatives (mothers and fathers of cases and controls) alone, patients with AGS were not significantly more likely to have a parent with a reported autoimmune disease $(\mathrm{OR}=2.24$; $P=0.29 ; 95 \%$ CI $=0.51-9.91)$. Of note, however, all of the parents who were reported to have autoimmune disease were in the US-based cohort of families (Table 1). If the US cohort is considered alone (Table 3), patients with AGS were statistically significantly more likely to have a parent with reported autoimmune disease $(\mathrm{OR}=5.09 ; P=0.040$; $95 \% \mathrm{CI}=1.07-24.02)$. Reports of autoimmune diseases in first degree relatives of children with AGS included diagnoses of: thyroid dysfunction, psoriasis, lupus-like symptoms, and ulcerative colitis.

Logistic regression was again used for second-degree relatives alone (grandparents, aunts, and uncles of patients (Table 2)). Patients with AGS were significantly more likely to have at least one second degree relative with autoimmune disease compared to controls $(\mathrm{OR}=2.88, P=0.044$,
95\% CI $=1.03-8.07$ ) (Table 2). A linear trend test was performed to determine if the odds ratio increased with increasing numbers of second degree relatives with reported autoimmune disease. There was evidence suggestive of a trend in the number of maternal family members $(P=$ 0.086 ), but not in the number of paternal family members. Reports of autoimmune disease diagnoses in this group of second-degree relatives of AGS included: chilblains, sarcoidosis, psoriasis, lupus, inflammatory bowel disease, Raynaud's, scleroderma, multiple sclerosis, thyroid dysfunction, and rheumatoid arthritis. Individual numbers were too small to make meaningful comments about subsets of autoimmune disorders, specifically SLE, seen in this population.

Because there was a difference in the report of autoimmune disease diagnoses between females (mothers) versus males (fathers), an assessment of differences in female versus male second-degree relatives' report of autoimmune disease diagnoses was performed. Among the 29 second-degree relatives in the AGS group who were reported to have autoimmune disease diagnoses, $21(72.4 \%)$ were female and $8(27.6 \%)$ were male. Among the 15 individuals in the control group who were reported to have an autoimmune disease diagnosis, $10(66.7 \%)$ were female and $5(33.3 \%)$ were male.

\section{Discussion}

Anecdotal evidence of an increased prevalence of autoimmune disease in family members of patients with AGS prompted this analysis of pedigrees of AGS patients in comparison to control families. AGS is part of a growing group of inherited disorders in which increased alpha interferon is thought to play a substantial role in the pathogenesis [21]. Familial increases in alpha interferon has been shown to aggregate with increased risk of established autoimmune 
TABLE 3: Comparison of autoimmune disease reported in first degree relatives in US-based cohort only.

\begin{tabular}{|c|c|c|c|c|c|}
\hline Characteristic & AGS cases & Control & OR & $P$ value & $95 \% \mathrm{CI}$ \\
\hline \multicolumn{6}{|c|}{ Maternal and/or paternal history of AI (yes/no) } \\
\hline No & $11(64.7 \%)$ & $28(90.3 \%)$ & 1.00 & & \\
\hline Yes & $6(35.3 \%)$ & $3(9.7 \%)$ & 5.09 & 0.040 & $1.07-24.02$ \\
\hline
\end{tabular}

disorders such as SLE [22] and juvenile dermatomyositis [23]. Thus, it is not surprising that there is a greater percentage of relatives of patients with AGS with reported autoimmune disease compared to controls, and that this association was statistically significant. It should be noted that this statistically significant association held for maternal relatives, but not so for paternal relatives. Patients with AGS in the US cohort alone were shown to be statistically significantly more likely to have a parent with autoimmune disease compared to controls.

To explain the difference between the US and Italian cohorts, genotype was considered, since a larger proportion of in the Italian group of AGS patients had mutations in $R N A S E H 2 B$, but removing those from the analysis did not result in any substantial change in findings. Thus, this difference is not understood and requires additional investigation. It is likely that the sample size is simply too small to detect meaningful differences.

Statistical analysis did show that children with AGS were significantly more likely to have at least one second degree relative with autoimmune disease as compared to controls. The finding of an increase in prevalence in females as compared to males was unexpected, although it is consistent with data showing that, in general, women are 2-3 times more likely to have autoimmune disease than men [18]. Although difficult to capture due to the variability of autoimmune diseases, the prevalence of autoimmune disease in the US may be approximately $1 / 31$ or $3.2 \%$ [18]. Our control population was found to have a slightly higher prevalence of autoimmune diseases, but our case population was found to have significantly more than this rate. The slight increase in prevalence in controls may be due to the fact that our controls were drawn from children with other neurogenetic diseases. Assessing differences in prevalence of autoimmune diseases in pedigrees of AGS families and pedigrees of families without any neurogenetic disease may provide a more accurate comparison.

It is unclear at this time whether the increased prevalence of autoimmune disease in relatives of AGS patients is directly related to mutation status in these relatives. Of note, although parents were generally presumed to be carriers of mutations in AGS-related genes, the genotype of second degree relatives with reported autoimmune disorders was not known. In addition, it is unknown whether other genetic modifiers exist that could change the phenotypic expression of mutations in AGS related genes, which may predispose related individuals to autoimmune diseases. Finally, other factors may exist, including the fact that families with a diagnosis of AGS may be more attentive to symptoms of autoimmune disorders than control families, or that other familial confounders may exist, such as diet, environment or infection. This may explain differences seen between the US and Italian cohorts, for example. Therefore, there is not enough evidence or understanding at this time to claim that individuals with diagnosed autoimmune diseases should be screened for mutations in AGS-related genes, or that family members of AGS patients known to carry a heterozygous mutation should be screened for autoimmune disorders.

However, it is of note that TREX 1 mutations have been found in patients with SLE. The relative risk for the development of SLE among those who carry TREX1 variants has been found to be 25.3 (95\% CI $=5.6-232.0)$ in one cohort $(N=317)$ [9]. In another recently studied large cohort of SLE patient $(N=8730)$, mutations in TREX1 one occurred at a frequency of $0.5 \%$ [10]. This data, in addition to our finding of increased prevalence of autoimmune disease in family members of AGS patients, suggests the need for further research into genotype-phenotype correlations of AGS related mutations and autoimmune disease.

\section{Limitations}

Because family history data was obtained by report of parents of cases and controls, and not by primary analysis of family members' medical records, data may be inaccurate in both cases and controls. It is possible that both families of patients with AGS and control patients experienced recall bias or that they were not aware of autoimmune diagnoses in the family, and, thus, prevalence of autoimmune disease in both groups may be underreported. Additionally, because the semistructured interviews for family history information were done at different times and by different investigators, not all cases and controls were asked about the same conditions in the same way, although every attempt was made to make the data as consistent as possible, including the use of the same group of investigators to collect control and AGS pedigrees.

One consanguineous case family and one consanguineous control family were included in the analysis. This was unlikely to have had an effect on the data, since second degree relatives of affected children were likely still only at 50\% risk of having inherited a heterozygous mutation from a parent. There were no reports of affected individuals in either the case or control family in previous generations.

AGS is a rare disorder, and therefore, sample sizes are small. However, the disease may be underdiagnosed. As awareness of AGS grows and additional patients are diagnosed, more family history data will be available. Also, as the understanding of the autoimmune nature of this disease improves, more robust conclusions can be drawn about the association and risk for developing autoimmune disease in obligate or presumed carriers. 


\section{Conclusion}

In this evaluation of family pedigrees from patients with Aicardi-Goutières Syndrome and control subjects, relatives of patients with AGS reported autoimmune disease diagnoses more frequently than family members of control patients; patients with AGS in the US-based cohort alone were statistically more likely to have a first-degree relative with an autoimmune disease and the entire cohort was found to be statistically more likely compared to controls to have at least one second-degree relative with autoimmune disease. Female relatives reported autoimmune disease diagnoses more frequently than male relatives. This data, in addition to the presumed autoimmune nature of AGS and the fact that genes that cause AGS (i.e., TREX1 and SAMHD1) are allelic to those that can cause SLE and FCL, warrants further study into the association between heterozygous mutations in AGS-related genes and autoimmune disease. There is not enough data to suggest that heterozygous carriers of mutations in AGS-related genes are at risk for developing autoimmune disease, nor that those individuals with autoimmune disease should be screened for mutations in these genes, but further work is needed to better understand the association.

\section{Acknowledgments}

We would like to thank the families for their cooperation in the work presented here. The research leading to these results has received funding from the European Union's Seventh Framework Program (FP7/2007-2013) under Grant no. 241779. The authors' would like to thank Dr. Yanick Crow for his guidance and leadership in efforts towards learning about AGS. They would also like to thank Dr. Miriam Bloom, Dr. Philip Pearl, Dr. Sally Evans, and Dr. Umberto Balottin for their help in evaluation of the patients and families studied.

\section{References}

[1] Y. J. Crow and J. H. Livingston, "Aicardi-Goutières syndrome: an important Mendelian mimic of congenital infection," Developmental Medicine and Child Neurology, vol. 50, no. 6, pp. 410-416, 2008.

[2] P. Lebon, J. Badoual, G. Ponsot, F. Goutieres, F. HemeuryCukier, and J. Aicardi, "Intrathecal synthesis of interferon- $\alpha$ in infants with progressive familial encephalopathy," Journal of the Neurological Sciences, vol. 84, no. 2-3, pp. 201-208, 1988.

[3] N. Blau, L. Bonafé, I. Krägeloh-Mann et al., "Cerebrospinal fluid pterins and folates in Aicardi-Goutières syndrome: a new phenotype," Neurology, vol. 61, no. 5, pp. 642-647, 2003.

[4] E. Wassmer, J. Singh, S. Agrawal, S. Santra, and Y. J. Crow, "Elevated pterins in cerebral spinal fluid-biochemical marker of Aicardi-Goutières syndrome," Developmental Medicine and Child Neurology, vol. 51, no. 10, pp. 841-842, 2009.

[5] J. Aicardi and F. Goutieres, "Systemic lupus erythematosus or Aicardi-Goutières syndrome?” Neuropediatrics, vol. 31, no. 3, article 113, 2000.

[6] Y. J. Crow, D. N. Black, M. Ali et al., "Cree encephalitis is allelic with aicardi-goutières syndrome: implications for the pathogenesis of disorders of interferon $\alpha$ metabolism," Journal of Medical Genetics, vol. 40, no. 3, pp. 183-187, 2003.
[7] C. de Laet, P. Goyens, C. Christophe, A. Ferster, F. Mascart, and B. Dan, "Phenotypic overlap between infantile systemic lupus erythematosus and Aicardi-Goutières syndrome," Neuropediatrics, vol. 36, no. 6, pp. 399-402, 2005.

[8] R. C. Dale, S. Ping Tang, J. Z. Heckmatt, and F. M. Tatnall, "Familial systemic lupus erythematosus and congenital infection-like syndrome," Neuropediatrics, vol. 31, no. 3, pp. 155-158, 2000.

[9] M. A. Lee-Kirsch, M. Gong, D. Chowdhury et al., "Mutations in the gene encoding the $3^{\prime}-5^{\prime}$ DNA exonuclease TREX1 are associated with systemic lupus erythematosus," Nature Genetics, vol. 39, no. 9, pp. 1065-1067, 2007.

[10] B. Namjou, P. H. Kothari, J. A. Kelly et al., "Evaluation of the TREX1 gene in a large multi-ancestral lupus cohort," Genes and Immunity, vol. 12, no. 4, pp. 270-279, 2011.

[11] C. Günther, M. Meurer, A. Stein, A. Viehweg, and M. A. Lee-Kirsch, "Familial chilblain lupus-a monogenic form of cutaneous lupus erythematosus due to a heterozygous mutation in TREX1," Dermatology, vol. 219, no. 2, pp. 162-166, 2009.

[12] G. Rice, W. G. Newman, J. Dean et al., "Heterozygous mutations in TREX1 cause familial chilblain lupus and dominant Aicardi-Goutières syndrome," American Journal of Human Genetics, vol. 80, no. 4, pp. 811-815, 2007.

[13] J. C. Ravenscroft, M. Suri, G. I. Rice, M. Szynkiewicz, and Y. J. Crow, "Autosomal dominant inheritance of a heterozygous mutation in SAMHD1 causing familial chilblain lupus," American Journal of Medical Genetics A, vol. 155, no. 1, pp. 235-237, 2011.

[14] A. Richards, A. M. J. M. van den Maagdenberg, J. C. Jen et al., "C-terminal truncations in human $3^{\prime}-5^{\prime}$ DNA exonuclease TREX1 cause autosomal dominant retinal vasculopathy with cerebral leukodystrophy," Nature Genetics, vol. 39, no. 9, pp. 1068-1070, 2007.

[15] D. C. Goldstone, V. Ennis-Adeniran, J. J. Hedden et al., "HIV-1 restriction factor SAMHD1 is a deoxynucleoside triphosphate triphosphohydrolase," Nature, vol. 480, no. 7377, pp. 379-382, 2011.

[16] G. I. Rice, J. Bond, A. Asipu et al., "Mutations involved in Aicardi-Goutières syndrome implicate SAMHD1 as regulator of the innate immune response," Nature Genetics, vol. 41, no. 7, pp. 829-832, 2009.

[17] D. B. Stetson, J. S. Ko, T. Heidmann, and R. Medzhitov, “Trex1 prevents cell-intrinsic initiation of autoimmunity," Cell, vol. 134, no. 4, pp. 587-598, 2008.

[18] D. L. Jacobson, S. J. Gangea, N. R. Roseb, and N. M.H. Graham, "Epidemiology and estimated population burden of selected autoimmune diseases in the United States," Clinical Immunology and Immunopathology, vol. 84, no. 3, pp. 223243, 1997.

[19] J. C. Prinz, "The role of T cells in psoriasis," Journal of the European Academy of Dermatology and Venereology, vol. 17, no. 3, pp. 257-270, 2003.

[20] J. C. Diniz, R. T. Almeida, N. E. Aikawa, A. Sallum, P. T. Sakane, and C. A. Silva, "Kawasaki disease and juvenile systemic lupus erythematosus," Lupus, vol. 21, no. 1, pp. 89-92, 2012.

[21] Y. J. Crow, "Type I interferonopathies: a novel set of inborn errors of immunity," Annals of the New York Academy of Sciences, vol. 1238, no. 1, pp. 91-98, 2011.

[22] T. B. Niewold, J. Hua, T. J. A. Lehman, J. B. Harley, and M. K. Crow, "High serum IFN- $\alpha$ activity is a heritable risk factor for systemic lupus erythematosus," Genes and Immunity, vol. 8, no. 6, pp. 492-502, 2007. 
[23] T. B. Niewold, S. C. Wu, M. Smith, G. A. Morgan, and L. M. Pachman, "Familial aggregation of autoimmune disease in juvenile dermatomyositis," Pediatrics, vol. 127, no. 5, pp. e1239-e1246, 2011. 


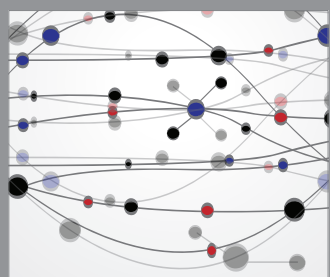

The Scientific World Journal
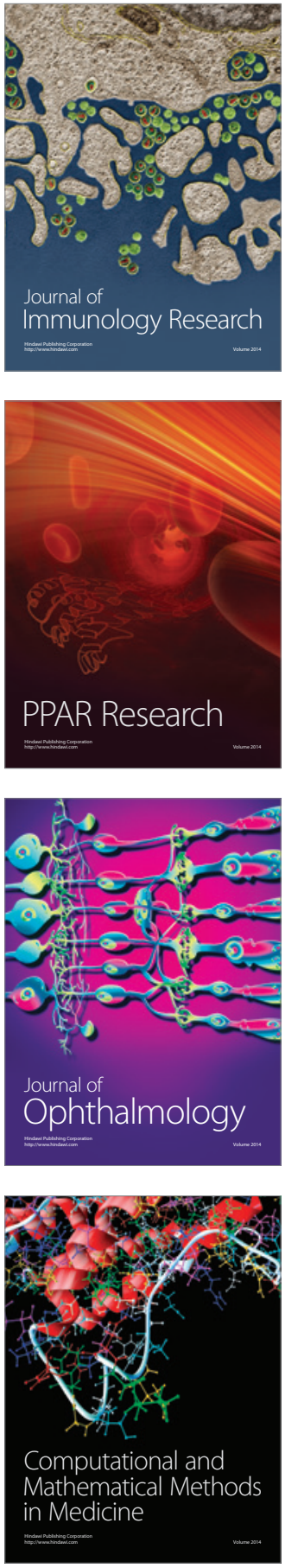



Gastroenterology

Research and Practice


\section{Hindawi}

Submit your manuscripts at

http://www.hindawi.com


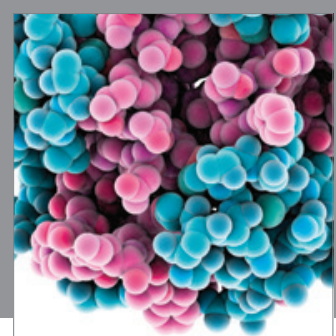

Journal of
Diabetes Research

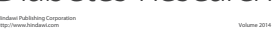



Disease Markers
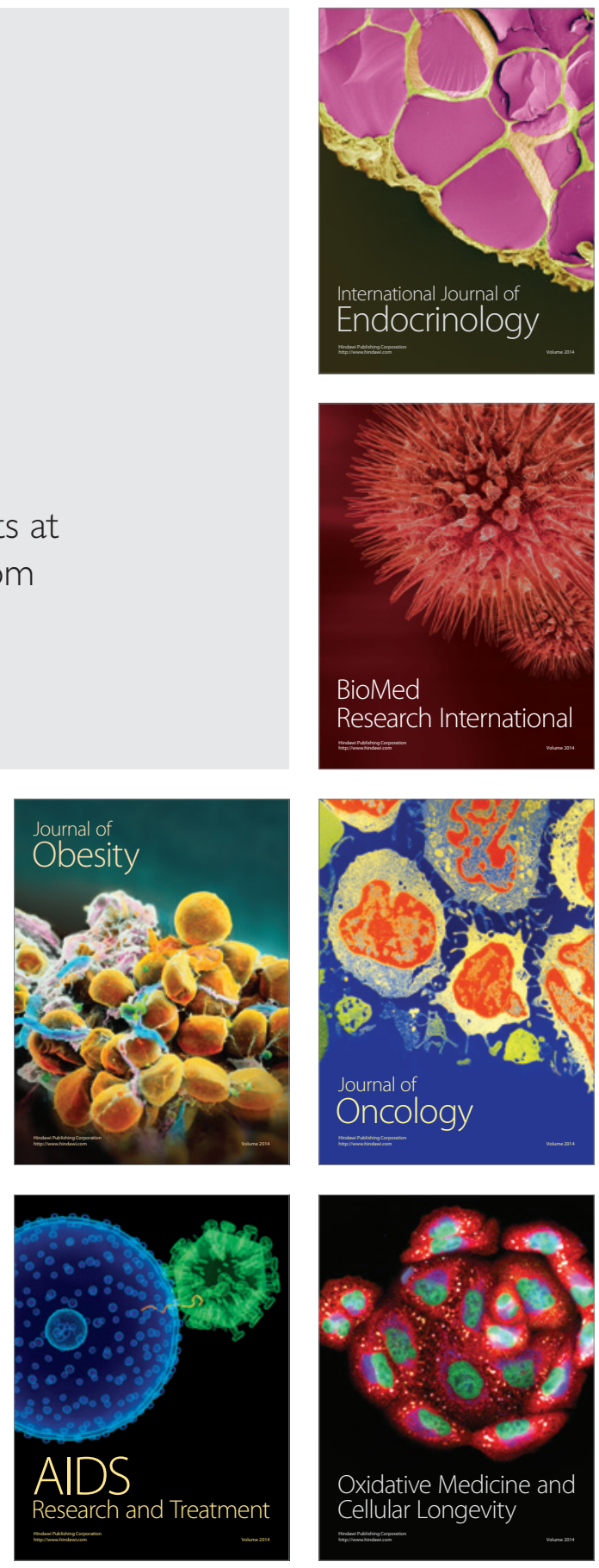